\title{
MINIMUM REDUNDANT APERTURE MASKING INTERFEROMETRY \\ WITH TIP-TILT WAVEFRONT CORRECTION
}

\author{
JUN NISHIKAWA \\ Hiraiso Solar Terrestrial Research Center, \\ Communications Research Laboratory, \\ S601 Isozaki, Nakaminato, Ibaraki \$11-12 Japan \\ YUTAKA HAYANO, NARUHISA TAKATO, MOTOKAZU NOGUCHI and \\ MASANORI IYE \\ National Astronomical Observatory, Mitaka, Tokyo 181 Japan \\ and \\ KOHHCHIRO MORITA and MASATO ISHIGURO \\ Nobeyama Radio Observatory, Minamimaki-mura, \\ Minamisaku-gun, Nagano 384-13 Japan
}

\begin{abstract}
A bstract. We made aperture masking optical interferometry experiments using up to $\mathbf{3 0}$ apertures and with a tip-tilt correction of wavefront error. We examined the performance of minimum redundant configurations of 11-30 sub-apertures on the pupil plane mask. These configurations have two advantages; the redundancy noise is as small as realized in non-redundant masking method, and the uv-coverage is as high as in speckle interferometry enabling to get reconstructed images without mask exchange. We also examined the effect of tip-tilt wavefront correction within a telescope pupil in front of the aperture masking optics. The light coherency between sub-apertures was shown to increase by the correction.
\end{abstract}

Key words: Aperture Synthesis - Interferometry - Speckle - Wavefront

\section{Minimum Redundant Configurations for Aperture Masking Method}

Redundant masking method takes an intermediate position between the speckle masking (SM) method (Lohmann et al. 1983) and the non-redundant masking (NRM) method (Haniff et al. 1987), and shares the advantages of the two methods. Minimum redundant masking (MRM) method is considered to be the most effective redundant masking method for imaging, which has following characteristics: 1 . High uv-coverage with a single exposure; 2 . A sufficient number of non-redundant baselines free from redundancy noise; 3 . Availability of visibility data at redundant baselines as constraint conditions in the Fourier space for mapping; 4. Attained limiting magnitude larger than by NRM, but smaller than by SM. MRM can produce enough Fourier components and their closure quantities by one exposure to reconstruct an image. It is possible to carry out image restoration not by point source fitting but by hybrid mapping method. If we use a few masks with different configuration, the reconstructed image can be obtained with much less ambiguity. When we fill up the Fourier space under a given observing time, small redundant configurations of many apertures with high uv-coverage, may be one of the best way to offer the longest integration time effective for each baseline, leading to high signal-to-noise ratio of interferometric data. The solar phenomena that varies as rapid as subseconds to minutes, are the suitable targets for the MRM. 
The performance of the MRM depends on the number and the distribution of sub-apertures within a primary mirror shaded by a secondary mirror. Fig. 1 shows minimum redundant configurations for 11 and 15 sub-apertures and near minimum redundant one for 30 sub-apertures. The sub-apertures are set on intersections of the grid. For the 15 sub-aperture configuration, non-redundant baselines cover $74 \%$ of the 184 Fourier points generated by the original full grid point distribution. Besides the square grid configurations, circular configurations with large odd numbers of apertures, multiple circular configurations, and $Y$-configurations are equally effective to get high uv-coverage with small redundancy, but they have systematic uncoverage or redundancy.

\section{Tip-Tilt Wavefront Correction in front of Aperture Masking Optics}

Without the low-order wavefront correction for aperture masking and speckle interferometry, the light coherency between distant sub-areas in a large telescope pupil decreases due to the optical delay. It is a severe problem for broad band observations under poor seeing conditions. The low-order wavefront correction will make fringes clearer and their motions slower on an imaging device.

We made experiments of aperture masking interferometry with a tip-tilt wavefront correction using $188 \mathrm{~cm}$ telescope at Okayama Astrophysical Observatory(OAO) and $91 \mathrm{~cm}$ at Dodaira Observatory(DO). Schematic diagram of our tip-tilt correction and aperture masking optics is shown in Fig. 2. The tip-tilt mirror was operated at the first pupil image to fix the second pupil image where an aperture mask was placed. This tip-tilt correction system was servo controlled at $200 \mathrm{~Hz}$ for a $3 m_{v}$ star. Our tip-tilt correction system has a performance to increase the peak intensity of a point source by 1.3 times when the seeing size was $1.3 \operatorname{arcsec}$ at $R$ band. Fig. 3 shows the mean Fourier power spectrum obtained by a point source $(\alpha$ Lyr) observation with the 15-aperture mask. Improvement on the Fourier power by applying the tip-tilt correction was confirmed, however the extent of improvement was not large enough to surpass the effect of variations of seeing condition. Fig. 4 shows the mean bi-spectrum for 455 triangles, the combinations of 3 apertures out of 15 , with and without the tip-tilt correction. The phase of the bi-spectrum, which indicates the closure phase of each triangle, is shown to concentrate close to zero when the tip-tilt correction is operated. These results indicate that the tip-tilt wavefront correction system with high throughput is effective to obtain clear fringe signals for aperture masking method.

\section{Acknowledgements}

We thank the staffs of OAO and DO for their support on our experiments, and Drs. N. Baba and H. Kobayashi for their helpful discussions.

\section{References}

Haniff, C.A.,Mackay, C.D.,Titterington, D.J., Sivia, D.,Baldwin, J.E.,and Warner, P.J.: 1987, Nature 328, 694.

Lohmann, A.W.,Weigelt, G., and Wirnitzer, B.: 1983, Appl. Opt. 22, 4028. 


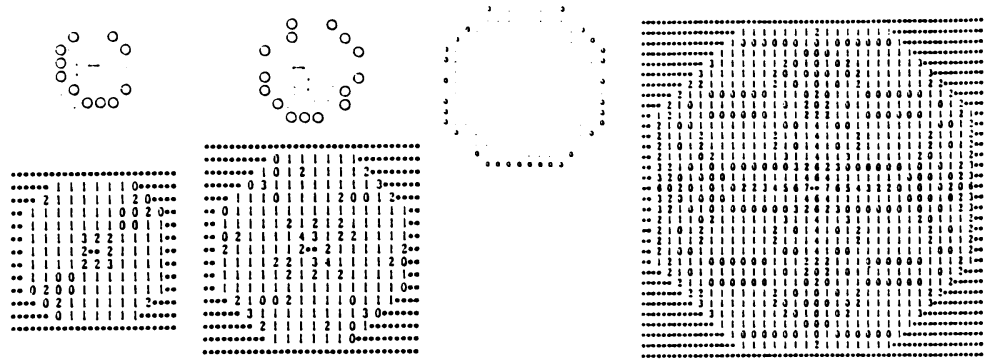

Fig. 1. Minimum redundant configurations for aperture masking method with 11,15 and 30 apertures. The upper(-left) panel for each configuration indicates the aperture distribution and the lower(-right) shows the distribution in the Fourier space. The number in the Fourier space shows redundancy at each point: 0 means no Fourier signal, 1 means non-redundant signal, 2 is double-redundant, and so on. The statistics for redundancy are as follows. For 11-aperture configuration, redundancy 0: 14(13\%) 1: 80(74\%) 2: 12(11\%) 3: 2(2\%); for 15 apertures, $0: 16(9 \%) 1: 136(74 \%) 2: 24(13 \%) 3: 6(3 \%) 4: 2(1 \%)$; and for 30 apertures, $0: 200(25 \%)$ 1: $464(57 \%)$ 2: $100(12 \%) 3: 26(3 \%) 4: 14(2 \%) 5-7: 12(1 \%)$.

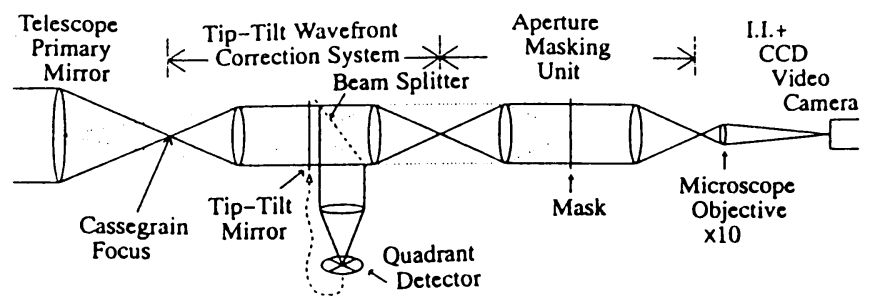

Fig. 2. Schematic diagram of the tip-tilt wavefront correction and aperture masking optics. The tip-tilt mirror and the aperture mask are placed at pupil images, respectively. Solid lines indicate the outline of optical path and the dotted lines show the ray through the center of telescope pupil. The tip-tilt mirror is controlled in two directions by piezoelectric translator to balance output signals from the quadrant photo diodes. Focal length of L1, $\mathrm{L} 2$, and L3 is $350 \mathrm{~mm}$, and L4 is $300 \mathrm{~mm}$. A beam-splitter picks up about a quarter of the light to the photo diode.

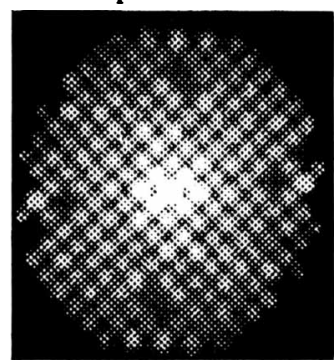

Fig. 3. Fourier power spectrum of a point source ( $\alpha$ Lyr) obtained with the minimum redundant 15-aperture mask. 2778 frames are averaged.
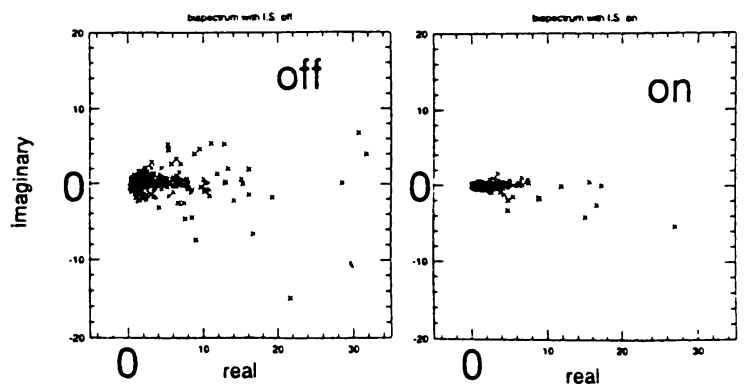

Fig. 4. Bi-spectrum of á point source $(\alpha$ Lyr $)$ for with and without the tip-tilt correction, obtained with the 15-aperture mask. Each cross indicates the complex value of the averaged bi-spectrum obtained from 2666-frame for a triangle. 